\title{
John Peter Pham, Heirs of the Fisherman. Behind the Scenes of Papal Death and Succession
}

New York, Oxford University Press, 2004, 368 p.

Ronan Teyssier

\section{(Q) OpenEdition}

\section{Journals}

Édition électronique

URL : http://journals.openedition.org/assr/22941

DOI : $10.4000 /$ assr.22941

ISSN : $1777-5825$

Éditeur

Éditions de l'EHESS

Édition imprimée

Date de publication : 31 décembre 2011

Pagination : 256

ISBN : 9782713223273

ISSN : 0335-5985

\section{Référence électronique}

Ronan Teyssier, « John Peter Pham, Heirs of the Fisherman. Behind the Scenes of Papal Death and Succession », Archives de sciences sociales des religions [En ligne], 156 | octobre-décembre 2011, document 156-91, mis en ligne le 16 février 2012, consulté le 21 septembre 2020. URL : http:// journals.openedition.org/assr/22941; DOI : https://doi.org/10.4000/assr.22941

Ce document a été généré automatiquement le 21 septembre 2020.

(c) Archives de sciences sociales des religions 


\section{John Peter Pham, Heirs of the Fisherman. Behind the Scenes of Papal Death and Succession}

New York, Oxford University Press, 2004, 368 p.

\section{Ronan Teyssier}

\section{RÉFÉRENCE}

John Peter Pham, Heirs of the Fisherman. Behind the Scenes of Papal Death and Succession, New York, Oxford University Press, 2004, 368 p.

1 Durant les premières années de la décennie 2000, alors que le pape Jean-Paul II était gravement malade, que la question de sa succession et celle de l'élection d'un nouveau pape devenaient de plus en plus prégnantes, nous avons assisté à l'essor des travaux historiques traitant de la succession et de la sélection des papes mais aussi à celui des récits de fiction abordant le thème et dont certains furent même portés à l'écran. Si l'ouvrage de John P. Pham s'inscrit pleinement dans ce contexte, il convient de préciser d'emblée que dans le travail recensé ici, l'auteur fait œuvre d'historien ayant eu accès à des témoins privilégiés au sein du Vatican (p.xiv). Comme le rapporte toutefois l'auteur, le secret entourant la succession des papes est si jalousement gardé qu'il nous faut tout de suite annoncer que ce livre ne dit pas tout sur la succession et la sélection des papes. Nombre d'aspects de cette question demeurent en effet secrets.

Le livre est composé de deux grands blocs dont seul le premier nous intéresse ici : il y a d'une part le texte à proprement parler qui représente un peu plus de cent soixante-dix pages et, d'autre part, les annexes qui représentent environ cent quarante pages. Ces dernières, volumineuses, servent bien le texte. Ainsi le lecteur soucieux d'approfondir un passage, de mieux comprendre une situation historique particulière ou encore un propos explicatif de l'auteur les consultera-t-il avec profit. 
3 Les six chapitres que comporte le livre peuvent être regroupés en trois parties. Par ordre d'importance vis-à-vis du sujet du livre, à savoir la succession des papes, on trouve premièrement une partie historique développée tout au long des chapitres 2, 3 et 4 ; ce travail d'historien est lui-même entouré d'une description des procédures et rituels entourant la mort des papes et leur succession (chap. 1 et 5). Puis J. P. Pham conclut sur une partie prospective (chapitre 6).

4 La partie historique comprend trois chapitres traitant respectivement de la désignation des papes avant que le conclave n'existe, de l'histoire du conclave en tant qu'institution électorale et enfin, des huit conclaves $\mathrm{du} \mathrm{xx}^{\mathrm{e}}$ siècle. Tout au long de ce parcours historique l'auteur met en évidence le caractère résolument dynamique et évolutif des procédures de désignation des papes. Si la désignation divine est évoquée rapidement, l'intérêt véritable du chapitre 2 est de montrer que l'important, pour l'Église, a rapidement été de trouver un mode de désignation des papes qui puisse prendre en compte la volonté du pape sortant tout en soustrayant la sélection du nouveau pape aux influences extérieures. Le moment crucial cristallisant ce mouvement historique est le décret du pape Nicolas II en 1059 (p. 58) en vertu duquel le résultat de l'élection papale ne devait désormais plus dépendre que du Collège des cardinaux. Cela signa en pratique la fin de l'influence impériale. L'autre moment important, qui a donné au conclave son nom (cum clave) et sa forme moderne, est intervenu au milieu du XIII ${ }^{\mathrm{e}}$ siècle. Il s'agit de la mise sous clé des cardinaux le temps que ces derniers parviennent à s'entendre sur l'élection d'un nouveau pape (p. 62). Cela donne une place centrale aux cardinaux que J.P. Pham décrit bien (p.63-76). Dans les pages 76-96, il détaille comment le conclave est organisé et comment les papes successifs ont affecté par leurs bulles le mécanisme électoral apportant quelques ajustements le plus souvent graduels à la structure mise en place en 1271 .

5 L'aspect véritablement intéressant dans ce parcours historique est la tentative présentée par l'auteur de tirer ce qu'il appelle des «leçons de l'histoire ». Ces leçons ont toutes pour base l'analyse des conclaves $d u x^{e}$ siècle. J.P. Pham retient tout d'abord que les considérations politiques jouent encore une part significative dans la désignation des papes même s'il ne s'agit peut-être pas de la part prédominante (p.132). Deuxièmement, lorsque les considérations politiques sont contrôlées, les considérations religieuses peuvent être plus importantes que les considérations temporelles. L'auteur cite en exemple le fait que, lors de l'élection papale de 1914, la question du modernisme fut plus déterminante que la situation politique et militaire internationale. Troisièmement, le nombre de cardinaux ayant augmenté au fil du temps, le pouvoir de blocage de chaque cardinal a été continuellement réduit. Finalement, il observe que les procédures électorales sont dynamiques et qu'elles changent. La meilleure illustration en est la bulle de Jean-Paul II Universi Dominici Gregis, appliquée pour la première fois lors de l'élection de Benoît XVI. J. P. Pham note à juste titre que tous les papes du $x x^{e}$ siècle, à l'exception de Jean-Paul $1^{\text {er }}$ qui n'est demeuré pape que quelques semaines, ont modifié les règles électorales présidant à la désignation de leur successeur.

6 On peut dire du cœur historique et descriptif du livre qu'il est placé entre deux chapitres (chap. 1 et 5) décrivant les rituels entourant la mort et la succession du pape. Ainsi le premier chapitre détaille-t-il la chaîne d'événements qui se succèdent lorsqu'un pape décède. L'auteur insiste sur ce qu'il y a de permanent, malgré quelques évolutions, dans les rituels de confirmation de la mort du pape sortant et de deuil. Il en 
va de même pour le très bref chapitre 5 qui traite de l'investiture du nouveau pape : l'auteur y passe en revue la séquence allant du couronnement à l'inaugu-ration.

7 La troisième partie thématique (chap. d'introduction et chap.6) se veut plus prospective. L'introduction et la conclusion tournent autour du pontificat de Jean-Paul II et de sa probable succession (à l'époque où Pham écrit en 2004). Le chapitre 6 est très intéressant à cet égard car l'auteur s'y livre à de prudents pronostics, d'ailleurs complétés par l'annexe 3.

8 En conclusion, le seul reproche que l'on puisse faire à cet ouvrage tient en fin de compte à ce que le sous-titre annonce peut-être plus que ce que l'auteur est réellement en mesure de présenter. Une fois achevée la lecture de cet ouvrage, si le lecteur a une bonne idée de l'histoire électorale de la papauté, les coulisses de l'élection des papes, et notamment des papes récents, lui restent en effet très largement inconnues.

Il semble néanmoins important de souligner que ce livre possède la qualité remarquable de rendre compte en détail de l'histoire de la succession des papes, qui court tout de même sur deux millénaires, dans des limites d'environ cent soixante-dix pages. J. P. Pham réussit donc le tour de force de présenter l'histoire de la désignation des papes de manière concise tout en offrant un propos dense non dénué de détails historiques. Si la lecture de cet ouvrage est grandement conseillée à tous ceux qui s'intéressent à l'Église catholique en tant qu'institution, elle l'est tout autant et plus généralement aux lecteurs intéressés par les processus électoraux entendus au sens large. 